\section{OPEN ACCESS}

Edited and reviewed by: Qiwei Zhang,

Southern Medical University, China

*Correspondence: Jaya Rajaiya

jaya_rajaiya@meei.harvard.edu

Specialty section:

This article was submitted to

Virology,

a section of the journal

Frontiers in Microbiology

Received: 25 October 2018 Accepted: 20 November 2018 Published: 04 December 2018

Citation:

Ismail AM, Lee JS, Lee JY, Singh G,

Dyer DW, Seto D, Chodosh J and

Rajaiya J (2018) Corrigendum:

Adenoviromics: Mining the Human Adenovirus Species D Genome.

Front. Microbiol. 9:3005.

doi: 10.3389/fmicb.2018.03005

\title{
Corrigendum: Adenoviromics: Mining the Human Adenovirus Species D Genome
}

\begin{abstract}
Ashrafali M. Ismail', Ji Sun Lee ${ }^{1}$, Jeong Yoon Lee ${ }^{1,2}$, Gurdeep Singh ${ }^{3}$, David W. Dyer ${ }^{4}$, Donald Seto ${ }^{5}$, James Chodosh ${ }^{1}$ and Jaya Rajaiya ${ }^{1 *}$

${ }^{1}$ Howe Laboratory, Massachusetts Eye and Ear, Harvard Medical School, Boston, MA, United States, ${ }^{2}$ Molecular Virology Laboratory, Korea Zoonosis Research Institute, Jeonbuk National University, Jeonju, South Korea, ${ }^{3}$ Department of Cell and Systems Biology, University of Toronto, Toronto, ON, Canada, ${ }^{4}$ Department of Microbiology and Immunology, University of Oklahoma Health Sciences Center, Oklahoma City, OK, United States, ${ }^{5}$ Bioinformatics and Computational Biology Program, School of Systems Biology, George Mason University, Manassas, VI, United States
\end{abstract}

Keywords: adenovirus, genome, evolution, transcription factor, interactome

\section{A Corrigendum on}

\section{Adenoviromics: Mining the Human Adenovirus Species D Genome}

by Ismail, A. M., Lee, J. S., Lee, J. Y., Singh, G., Dyer, D. W., Seto, D., et al. (2018). Front. Microbiol. 9:2178. doi: 10.3389/fmicb.2018.02178

In the original article, there were some minor errors in Tables 1, 2 as published. Some HAdV species names were wrongly spelled and some species names were left out. In addition, we have also updated the correct year of publication for these types. The corrected Tables 1, 2 appear below. The authors apologize for this error and state that this does not change the scientific conclusions of the article in any way. The original article has been updated.

Conflict of Interest Statement: The authors declare that the research was conducted in the absence of any commercial or financial relationships that could be construed as a potential conflict of interest.

Copyright () 2018 Ismail, Lee, Lee, Singh, Dyer, Seto, Chodosh and Rajaiya. This is an open-access article distributed under the terms of the Creative Commons Attribution License (CC BY). The use, distribution or reproduction in other forums is permitted, provided the original author(s) and the copyright owner(s) are credited and that the original publication in this journal is cited, in accordance with accepted academic practice. No use, distribution or reproduction is permitted which does not comply with these terms. 
TABLE 1 | Species and type designations for the 51 human adenovirus (HAdV) serotypes.

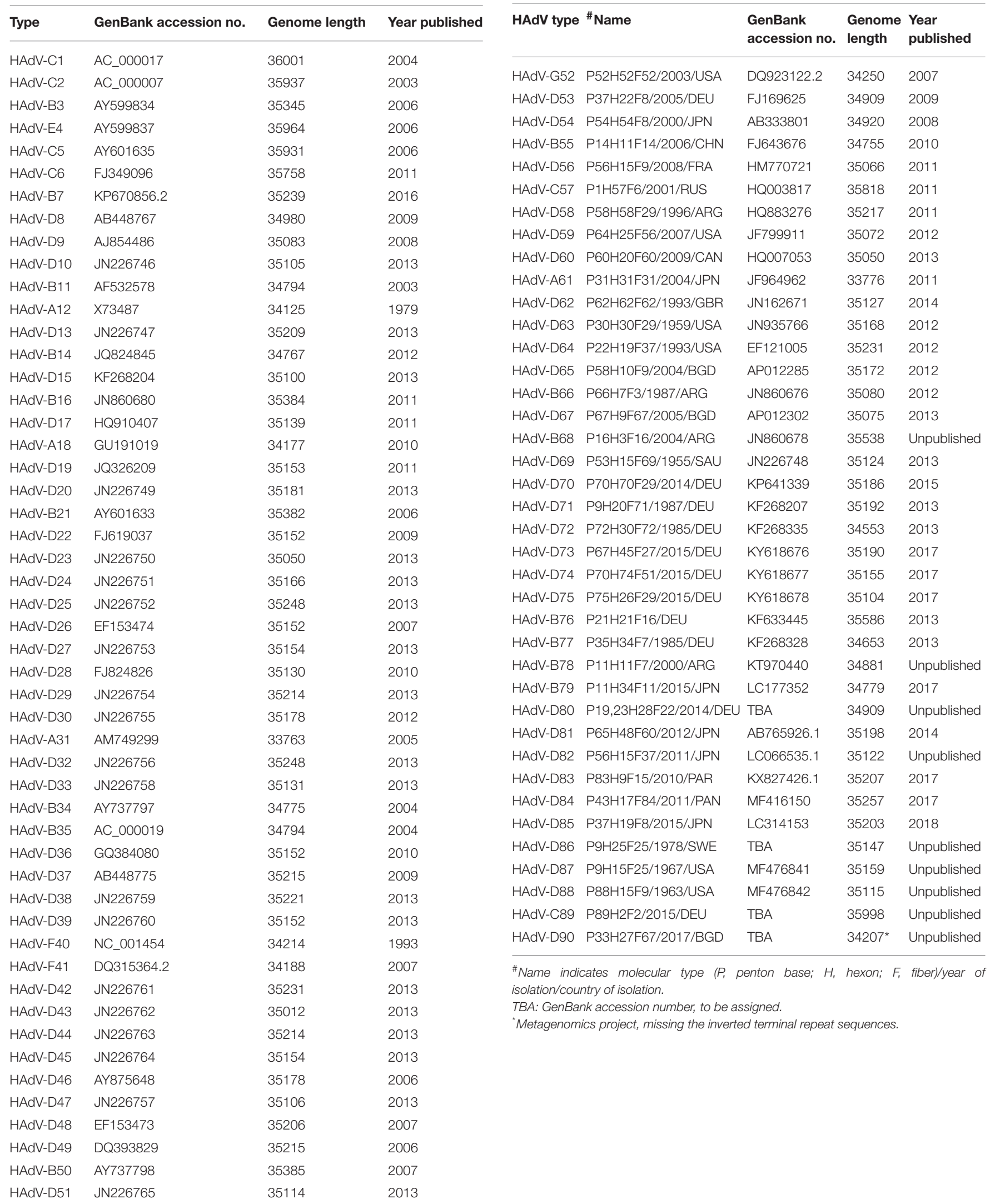

TABLE 2 | Species and molecular types of human adenovirus (HAdV) genotypes $52-90$.

$\begin{array}{lllll}\text { HAdV-D54 P54H54F8/2000/JPN } & \text { AB333801 } & 34920 & 2008\end{array}$

B55 $\mathrm{P} 14 \mathrm{H} 11 \mathrm{~F} 14 / 2006 / \mathrm{CHN} \quad \mathrm{FJ} 643676$

HAdV-D60 P6OH2OF60/2009/CAN HQ007053 350502013

HAdV-D64 P22H19F37/1993/USA EF121005 $35231 \quad 2012$

HAdV-D65 P58H10F9/2004/BGD AP012285 $35172 \quad 2012$

HAdV-B66 P66H7F3/1987/ARG JN860676 350802012

HAdV-D67 P67H9F67/2005/BGD AP012302 350752013

HAdV-B68 P16H3F16/2004/ARG JN860678 35538 Unpublished

HAdV-D75 P75H26F29/2015/DEU KY618678 351042017

HAdV-B76 P21H21F16/DEU $\quad$ KF633445 355862013

HAdV-B77 P35H34F7/1985/DEU KF268328 346532013

HAdV-B78 P11H11F7/2000/ARG KT970440 34881 Unpublished

HAdV-B79 P11H34F11/2015/JPN LC177352 347792017

HAdV-D80 P19,23H28F22/2014/DEU TBA 34909 Unpublished

HAdV-D81 P65H48F60/2012/JPN AB765926.1 $35198 \quad 2014$

HAdV-D82 P56H15F37/2011/JPN

AdV-D86 P9H25F25/1978/SWE

HAdV-D87 P9H15F25/1967/USA

HAdV-C89 P89H2F2/2015/DEU TBA 35998 Unpublished TBA: GenBank accession

TBA: GenBank accession number, to be assigned.
Metagenomics project, missing the inverted terminal repeat sequences. 